\title{
Evaluación de las condiciones de manejo de catéteres vasculares periféricos en pacientes adultos
}

\author{
Elena Véliz, Teresa Vergara y Alberto Fica
}

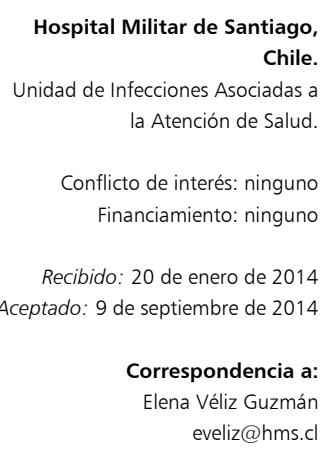

Correspondencia a: eveliz@hms.cl

\section{Evaluation of peripheral vascular catheter management conditions in adult patients}

Background: Peripheral venous vascular catheters (PVC) are devices that are not free of complications, and $\mathrm{CDC}$, Atlanta, recommendations are followed in order to prevent them. However, available information in Chile on this matter is scarce. Objective: To evaluate if management of PVC fulfill safety recommendations in adult patients in a general hospital. Methods: Cross-sectional study evaluating compliance with 4 measures: patch condition, circuit condition, justification of PVC and registration of the device on medical records. Results: We evaluated 102 PVC, and 45.1\% met all the measures assessed. Deficiencies were identified in $54.9 \%$ and included shortfalls in every one of the 4 topics measured. Compliance among those with iv sets was higher in comparison with those using mechanical valve needleless connectors $(2.5$ vs 1.9 points in a scale from 0 to $4 ; \mathrm{p}<0.001)$. In addition, compliance decreased progressively along time of use (Pearson coefficient $-0.4 ; \mathrm{p}<0.05)$. Conclusions: Deficiencies on the safety management of PVC were frequent in clinical practice and worsen with days. Needleless mechanical connectors use is associated with a lower compliance.

Key words: Peripheral vascular catheters, safe handling, phlebitis.

Palabras clave: catéteres vasculares periféricos, manejo seguro, flebitis.

\section{Introducción}

L os catéteres vasculares periféricos (CVP) son dispositivos útiles y de fácil instalación, pero no están libres de complicaciones como flebitis, bacteriemias, sepsis u obstrucción. Aunque estas complicaciones son de baja incidencia, son eventos perceptibles y cotidianos en la práctica asistencial y cuando se presentan originan importantes molestias al enfermo, pueden prolongar la estancia hospitalaria y también aumentar el gasto sanitario. ${ }^{1}$ Para prevenir estas complicaciones, los equipos de salud han usado las recomendaciones emanadas desde los Centers for Disease Control and Prevention (CDC) de Atlanta, EUA. ${ }^{2}$ Sin embargo, la información disponible en Chile sobre el manejo de los CVP es fragmentaria y restringida a neonatología ${ }^{3}$. Para aportar en el conocimiento del manejo de los CVP en nuestro medio, se efectuó una evaluación de las condiciones de seguridad de estos dispositivos en pacientes adultos en un hospital general. Sus resultados permiten poner en perspectiva posibles cambios en las recomendaciones sobre la duración de los CVP que han surgido recientemente en la literatura médica $^{4-7}$.

\section{Material y Método}

Se realizó un estudio transversal sobre el manejo de los CVP en un hospital de alta complejidad de Santiago de Chile. El estudio se efectuó durante un día en el mes de julio del 2013. Se incluyeron pacientes adultos hospitalizados en la Unidad de Pacientes Críticos (UPC), Servicio Médico-Quirúrgico o en la Unidad de HematoOncología y que estuvieran usando al menos un CVP el día del estudio. Se efectuó una inspección visual directa de los CVP de los pacientes y se revisaron además, los registros clínicos.

Los objetivos fueron evaluar el grado de cumplimiento seguro del CVP, ver diferencias entre CVP con o sin conectores con válvula mecánica, analizar diferencias por servicios y según tiempo de permanencia.

Para el manejo seguro se analizaron los siguientes aspectos:

- Condición del parche o apósito: Se consideró una condición segura si el parche cumplía el criterio de estar limpio, seco y adherido.

- Condición del circuito: En caso de conexión a equipo de fleboclisis, se consideró adecuado si éste estaba visiblemente limpio. No se evaluó este aspecto en presencia de conectores.

- Indicación del CVP. Este aspecto fue evaluado en base al cumplimiento de criterios de necesidad tales como: indicación médica de administración de medicamento o fluido endovenoso, examen con medio de contraste y/o trasfusión. Se consideró inadecuada si no había indicación vigente alguna. 
- Registro. Se consideró este criterio si había registro en relación a las condiciones del CVP dentro de las $12 \mathrm{hrs}$ previas al estudio, donde al menos se debía mencionar la existencia del CVP. La permanencia del CVP fue evaluada pero no fue incluida como criterio de seguridad ${ }^{4-7}$.

Las medidas consideradas seguras en este trabajo están basadas en las recomendaciones de los $\mathrm{CDC}^{2}$. Para tal efecto se recomienda la mantención en el sitio de inserción de un apósito estéril seco, limpio y sellado (evidencia IA). Por otro lado, los equipos de infusión deben ser cambiados a plazo fijo ya que éstos se colonizan con facilidad, más aún cuando se infunden soluciones con lípidos o sangre (IB); de ahí la importancia de evaluar las condiciones de limpieza visible de los equipos de infusión. El registro del estado del acceso vascular por parte del operador es necesario en el contexto de disponer de un verificador de la evolución del estado del acceso vascular y las actividades de mantención realizadas en éste (Categoría II). Por último, se requiere evaluación periódica de la necesidad del acceso vascular con el fin de suspender la indicación cuando éste ya no se requiera (Categoría IB $)^{2}$. Además se evaluó la presencia de flebitis como complicación asociada al CVP, considerando la clasificación de Jackson ${ }^{8}$.

\section{Análisis estadístico}

Los datos se presentan en forma descriptiva. Las diferencias fueron analizadas por prueba t-de Student o $\chi^{2}$ según el caso. Se aceptó un nivel de significación estadística $\mathrm{p}<0,05$.

\section{Resultados}

El día del estudio se encontraban hospitalizados 213 pacientes en los servicios clínicos involucrados y de ellos, 45\% (96 pacientes) tenía uno o más CVP con un total de 102 accesos periféricos. De los 102 pacientes con CVP evaluados, $49 \%$ (n: 50 ) se concentró en el Servicio de Medicina, luego Cirugía con 34,3\% (n: 35), UPC 15,7\% (n: 16) y Hemato-Oncología 1\% (n: 1). La menor prevalencia de CVP en UPC se explica por el predominio de accesos vasculares centrales.

Se contó con información sobre la permanencia del CVP en 98 casos (96,7\%). El promedio de permanencia fue 1,9 días (mediana 1 día; rango 0 a 11 días y percentil 75 de 3 días). Un 88,2\% (n: 90) tenía un tiempo de permanencia $\leq 96 \mathrm{~h}$. La permanencia del CVP no fue significativamente diferente entre los pacientes hospitalizados en UPC versus otro tipo de unidades de hospitalización ( 1,75 vs 1,9 días, respectivamente, $p>0,05$ por prueba $t$ de Student). En contraste, la permanencia del CVP fue

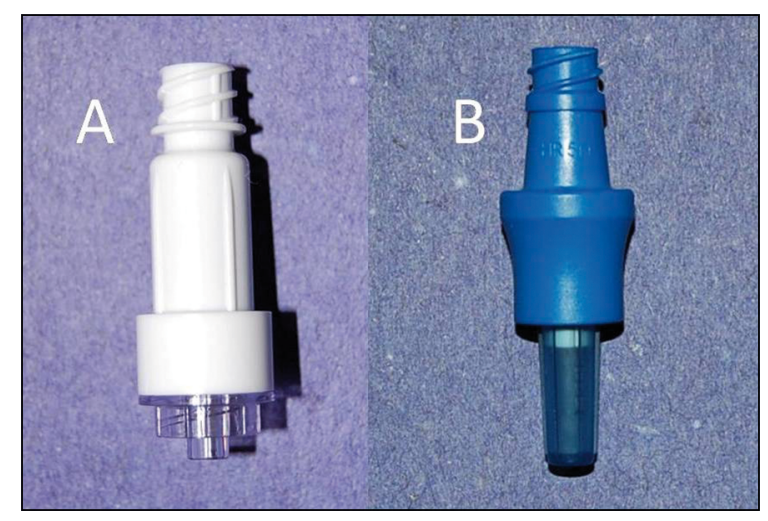

Figura 1A. Conector sin aguja de tercera generación con válvula mecánica de presión positiva. 1B. Conector de segunda generación de septum tabicado. Hacia arriba de la figura la zona de conexión con los sets de infusión.

significativamente más prolongada entre los pacientes del Servicio de Medicina respecto al Servicio de Cirugía (2,3 vs 1,4 días, $\mathrm{p}<0,05$ por prueba t de Student).

De los 102 CVP observados, 45,1\% (n: 46) cumplía con todas las medidas consideradas para un manejo seguro; sin embargo, el 54,9\% restante (n: 55) presentó incumplimiento en uno o más de los criterios establecidos. El porcentaje de cumplimiento no fue significativamente diferente en la UPC respecto los Servicios de Medicina o Cirugía $(37,5$ vs 46,5\%, $\mathrm{p}>0,05)$ ni entre los Servicios de Medicina o Cirugía (38 vs 57,1\%, p >0,05).

El día del estudio se identificó además un caso de flebitis (1\%) en un CVP de un día de permanencia que se encontraba sellado con un conector de válvula mecánica justificado. Sin embargo, no existía registro de enfermería acerca del estado del catéter en las últimas $12 \mathrm{~h}$.

La mayor parte de los CVP tenía circuito para fleboclisis (n: 72, 70,6\%) y la fracción restante $(29,4 \%, \mathrm{n}: 30)$ tenía conectores con válvula mecánica (Figura 1A) que no poseen un sistema continuo de infusión sino que son usados según necesidad.

Al realizar análisis del cumplimiento para cada una de las cuatro medidas evaluadas, se observaron brechas importantes en el manejo seguro y ningún componente con porcentajes elevados de cumplimiento (Tabla 1). Por ejemplo, se identificaron deficiencias en el estado del parche o apósito y en la justificación del uso del CVP en

Tabla 1. Cumplimiento por componentes en mantención de catéter venoso periférico (CVP)

\begin{tabular}{lcc} 
Componente & n/N & $\%$ \\
Parche o apósito adecuado & $83 / 102$ & 81,4 \\
Necesidad de CVP & $79 / 102$ & 77,5 \\
Circuito de fleboclisis limpio** & $66 / 72$ & 70,6 \\
Registro & $72 / 102$ & 70,6 \\
\hline *n/N: Número que cumplen componentes de mantención de CVP/ Número total de observaciones ; \\
**Sólo para CVP sin conectores.
\end{tabular}


cerca de $20 \%$ y por otra parte, se encontraron restos de sangre en el equipo de fleboclisis y registro inadecuado de las condiciones o de la mera existencia del CVP en $30 \%$ de los casos.

Aplicando una escala binaria (1: cumplimiento, 0: no cumplimiento) para cada una de las cuatro medidas consideradas en el manejo seguro del CVP (Tabla 1), se analizó el comportamiento de la suma total según la permanencia del CVP. Excluyendo los CVP con conectores con válvula mecánica que no tienen equipo de fleboclisis permanente que evaluar, se observó una reducción significativa en el grado de cumplimiento a medida que transcurre el tiempo del CVP (Figura 2, coeficiente de correlación de Pearson $-0,40 p<0,05)$. Por otra parte, excluyendo del análisis la variable fleboclisis (no presente en el grupo con conectores con válvula mecánica) para hacer comparables los grupos, el puntaje observado en los CVP sin estos conectores fue significativamente superior respecto al medido en aquellos con este tipo de conexiones (2,5 vs 1,9 de una escala máxima de 3 puntos, $\mathrm{p}<0,001$ por prueba t de Student, Figura 3). Las diferencias residen en dos de los tres aspectos evaluados: condición del parche o apósito

Figura 2. Nivel de cumplimiento de las medidas para un manejo seguro de CVP según permanencia. Escala 0 a 4 puntos. Se excluyeron pacientes con CVP y conectores con válvula mecánica.

Figura 3. Nivel de cumplimiento de las medidas para un manejo seguro de CVP según uso o no uso de conectores con válvula mecánica. Escala 0 a 3 puntos. Se excluyó del análisis la variable fleboclisis limpia para hacer los grupos comparables.
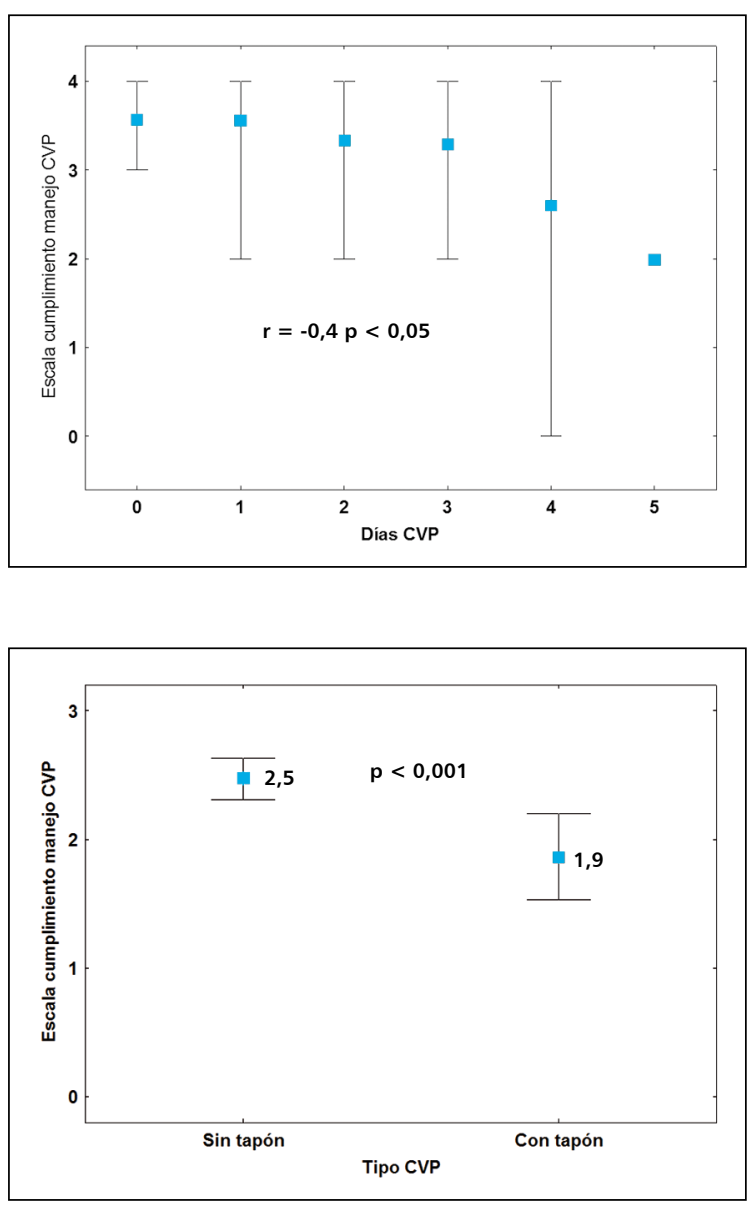

( 88,9 vs $63,3 \%$ de cumplimiento, $\mathrm{p}<0,01)$ y registro de las condiciones del CVP (76,4 vs 56,7\% de cumplimiento, $\mathrm{p}<0,05$ por prueba de $\chi^{2}$ ) (Figura 2).

\section{Discusión}

En esta investigación se pudieron constatar varias deficiencias en el manejo seguro del CVP que son importantes en la atención diaria de los pacientes: problemas en el proceso diario de supervisión sobre las condiciones del parche, sets de infusión y la necesidad real de un acceso periférico junto al deterioro progresivo del proceso de supervisión a medida que pasan los días y la sensación de falsa seguridad que rodea el uso de conectores con válvula mecánica. Como vimos, las deficiencias fueron prevalentes y bordearon el 50\% de los accesos. No encontramos estudios que evaluaran la calidad del manejo de CVP utilizando las mismas variables de nuestro estudio, pero otras investigaciones también han demostrando un cumplimiento deficiente en el manejo de CVP. ${ }^{9}$ Además, el conocimiento que el personal de enfermería tiene sobre el acceso vascular periférico, indica que sólo la mitad sabe que debe usar técnica aséptica al momento del cambio ${ }^{10}$. En nuestro trabajo, la frecuencia de flebitis fue baja respecto a lo observado en otros estudios, lo que se explica por su diseño de tipo transversal y no de incidencia. No se incluyó en este estudio una evaluación sobre el reemplazo de CVP a plazos preestablecidos debido a que ha sido una política cuestionada por diferentes estudios observacionales, randomizados o revisiones sistemáticas que señalan que el riesgo de flebitis, obstrucción o bacteriemia por CVP no aumenta al prolongar el uso del $\mathrm{CVP}^{4-7}$. La decisión de no cambiar los CVP a plazos preestablecidos tiene múltiples beneficios que incluyen disminuir la carga laboral, evitar molestias al paciente y disminuir los costos por insumos ${ }^{4-7,11}$.

Los resultados obtenidos en este trabajo deben ser confirmados en otros centros, pero dan pie a la implementación de un programa de intervención que aborde las falencias pesquisadas incluyendo la supervisión frecuente y la capacitación del equipo de salud, estrategias que han demostrado ser útiles para prevenir infecciones. ${ }^{2,4,10,12,13 .} \mathrm{La}$ auto-aplicación de una lista de chequeo de instalación y manejo de CVP también puede ser implementada como otra estrategia de intervención ${ }^{14}$.

Los conectores sin aguja fueron introducidos para disminuir el riesgo de accidentes cortopunzantes en el personal de salud ${ }^{15,16}$. Actualmente se encuentran ampliamente disponibles los conectores con septum tabicado (split septum) considerados de segunda generación (Figura 1B) o los conectores de válvula mecánica (mechanical valve connectors) denominados de tercera generación, que se caracterizan por tener intrincados diseños internos con mecanismos de resortes y presiones neutras, positivas 
o negativas (Figura 1A). Estos conectores (segunda o tercera generación) han ganado aceptación también por la facilidad de establecer las infusiones y conexiones. En nuestro hospital se incorporaron conectores de válvula mecánica de presión positiva y el uso de este tipo de conexiones se asoció a una menor vigilancia, la que fue progresiva en el tiempo y probablemente explicada por una falsa sensación de seguridad. Aunque no se han asociado a una mayor tasa de flebitis, sí se han relacionado a una mayor tasa de bacteriemias por CVC respecto a conectores con septum tabicado ${ }^{15,16}$. Lamentablemente, la capacitación del personal de salud no incide mucho en disminuir las bacteriemias asociadas a su uso y en muchos lugares se ha optado por su retiro ${ }^{15,16}$. Este tipo de conexiones podría así incrementar el riesgo de flebitis o de bacteriemia si se disminuye la frecuencia en el cambio del CVP. Así, una combinación de baja supervisión con un diseño inadecuado podría resultar de mayor riesgo al espaciar los cambios de los CVP.

\section{Resumen}

Introducción: Los catéteres vasculares venosos periféricos (CVP) son dispositivos que no están libres de complicaciones. Para prevenir estas complicaciones, los equipos de salud han usado las recomendaciones emanadas de los Centers for Disease Control and Prevention (CDC) de Atlanta, EUA. Sin embargo, la información disponible en Chile sobre el manejo de los CVP es fragmentaria. Objetivo: Evaluar las condiciones de seguridad de estos dispositivos en pacientes adultos en un hospital general. Material y Método: Estudio transversal con evaluación de CVP según cumplimiento de cuatro medidas: condición del parche, condición del circuito, justificación del uso del CVP y registro apropiado. Resultados: Se evaluaron 102 CVP. Sólo el $45,1 \%$ cumplía con todas las medidas evaluadas. Se encontraron deficiencias en las cuatro medidas evaluadas. El cumplimiento en CVP con circuito de fleboclisis fue superior respecto a aquellos con conectores con válvula mecánica $(2,5$ vs 1,9 puntos en una escala de 0 a 4 puntos, $\mathrm{p}<0,001)$. Se observó reducción en el grado de cumplimiento a medida que trascurre el tiempo del CVP (coeficiente de Pearson -0,4; $<<0,05$ ). Conclusiones: Se constataron deficiencias frecuentes en el manejo de los CVP, las que empeoran a medida que pasan los días. El uso de conectores mecánicos se asocia a una disminución del proceso de supervisión.

\section{Referencias bibliográficas}

1.- Ferrete C, Vásquez M, Sánchez M, Gilabert I-Cerro, Corzo J, Pineda J, et al. Incidencia de flebitis secundaria por catéter venoso de acceso periférico e impacto de un protocolo de manejo. Enfermería clínica 2010; 20: 3-9.

2.- CDC. Guidelines for the Prevention of Intravascular Catheter-Related Infections, 2011 Disponible en http://www.cdc.gov/hicpac/pdf/ guidelines/bsi-guidelines-2011.pdf (accedido 12 diciembre 2013)

3.- Barría M, Santander G. Acceso vascular periférico en neonatos de cuidado intensivo: experiencia de un hospital público. Ciencia y Enfermería. 2006; XII: 35-44.

4.- Webster J, Osborne S, Rickard C, New K. Clinically-indicated replacement versus routine replacement of peripheral venous catheters. Cochrane Database Syst Rev 2013; 4:CD007798.

5.- Bregenzer T, Conen D, Sakmann P, Widmer A. Is routine replacement of peripheral intravenous catheters necessary? Arch Intern Med. 1998; 158: 151-6.

6.- Rickard C, McCann D, Munnings J, McGrail M.
Routine resite of peripheral intravenous devices every 3 days did not reduce complications compared with clinically indicated resite: a randomised controlled trial. BMC Med. 2010; 8: 53.

7.- Rickard C, Webster J, Wallis M, Marsh N, McGrail M, French V, et al. Routine versus clinically indicated replacement of peripheral intravenous catheters: a randomised controlled equivalence trial. Lancet 2012; 380: 1066-74.

8.- Jackson A. Infection control-a battle in vein: infusion phlebitis. Nurs Times 1998; 3 : 68-71.

9.- Yassuko A, Gomes da Silva G, Bellucci J, Camboin V, Willamowius D, Misue L. Evaluation of care in intravenous therapy: a challenge for quality in nursing. Esc Anna Nery 2013; 17: 11-16.

10.- Cicolini G, Simonetti V, Comparcini D, Labeau S, Blot S, Pelusi G, et al. Nurses' knowledge of evidence-based guidelines on the prevention of peripheral venous catheter- related infections: A multicentre survey. J Clin Nurs 2013; 23: 2578-88.

11.- Cornely OA, Bethe U, Pauls R, Waldschmidt D. Peripheral teflon catheters: factors determining incidence of phlebitis and duration of cannulation. Infect Control Hosp Epidemiol 2002; 23: 249-53.

12.- García I, Gálvez M, Barrio J. Estudio de la incidencia de flebitis en enfermos portadores de catéteres venosos periféricos (CVP). Disponible en http://www.fac.org.ar/tcve/llave/t1058/t1058. PDF. (accedido 12 diciembre 2013).

13.- Campbell L. I.v.-related phlebitis, complications and length of hospital stay: 1. Br J Nurs 1998; 7: 1304-6. 1308-12.

14.- Sebastián T, Núñez F, Martín G, González J, Lema I, Salvadores P, Losa M. Impacto de la implantación de recordatorios para disminuir eventos adversos en pacientes con accesos venosos periféricos. An Sist Sanit Navar 2012; 35: 395-402.

15.- Jarvis W R, Murphy C, Hall K K, Fogle P J, Karchmer T B, Harrington G, et al. Health care-associated bloodstream infections associated with negative- or positive-pressure or displacement mechanical valve needleless connectors. Clin Infect Dis 2009; 49: 1821-7.

16.- Btaiche I F, Kovacevich D S, Khalidi N, Papke L F. The effects of needleless connectors on catheter-related bloodstream infections. Am J Infect Control 2011; 39: 277-83. 